\title{
A THERMOPNEUMATICALLY-ACTUATED MICROVALVE WITH IMPROVED THERMAL EFFICIENCY AND INTEGRATED STATE SENSING
}

\author{
Collin A. Rich ${ }^{*}$ and Kensall D. Wise \\ Department of EECS, University of Michigan \\ Ann Arbor, MI 48109-2122
}

\begin{abstract}
A thermopneumatically-actuated microvalve that is suitable for integration into batch-fabricated microsystems is reported. The valve achieves a $420 \mathrm{sccm}$ open flow under 1500torr differential and $<0.01 \mathrm{sccm}$ leak rate, yielding a dynamic range $>4 \times 10^{4}$. The saturated vapor pressure of a resistively heated working fluid deflects a corrugated silicon valve plate to close the valve. The heater grids are elevated above the substrate and the cavity is only partially filled to increase thermal efficiency. An integral capacitive pressure sensor allows direct monitoring of actuator pressure. Pentane-filled actuators sustain a 2070torr pressure rise with $500 \mathrm{~mW}$. A device tested in situ closes with $350 \mathrm{~mW}$ at 1000torr inlet (venting to vacuum) and maintains closure with $30 \mathrm{~mW}$ input. Thermodynamic modeling matches experimental power, pressure, and transient response data to within a few percent. This model is used to suggest an optimized structure capable of a 2000 torr pressure rise with $50 \mathrm{~mW}$ input and a $1 \mathrm{sec}$ response time.
\end{abstract}

\section{INTRODUCTION}

Microfluidic systems constitute a steadily growing sector of the MEMS field. Potential applications include microreaction chambers, implantable drug delivery systems, "intelligent" petri dishes for microanalysis, a compact gas microchromatograph, and miniaturized mass flow controllers for precision applications. A suitable microvalve for such systems will have a wide dynamic range, low leak rate, fast response, and low power consumption. Additionally, compatibility with batch fabrication is important for developing integrated microfluidic systems.

This paper reports a thermopneumatically-actuated microvalve with a simple, glass-and-silicon composition that is suitable for integration into batch-fabricated microsystems. Thermopneumatic actuation was chosen for its robustness and ability to generate large forces over considerable distances, facilitating a valve with substantial dynamic range and low leak rate. Furthermore, response time and power requiremenls can be minimized with thermal optimization of the valve structure.

\section{DEVICE STRUCTURE}

The basic microvalve (see Fig. 1) comprises a silicon microstructure capped above and below by anodically-bonded glass plates. The valve diaphragm in the $1^{\text {st }} \mathrm{Si}$ bulk consists of a central boss supported by a corrugated suspension. In the open state, gas flow is determined by the inlet and outlet pressures, along with the dimensions of the flow channel. To close the valve, the diaphragm is deflected to occlude the gas inlet. The $1^{\text {st }} \mathrm{Si}$ fabrication process, reported elsewhere [1], uses an RIE etch to define the gas flow channel and valve plate corrugation profile.
Diffused boron etch-stops define the rim, boss, and corrugation thickness; an EDP etch step then releases the structure.

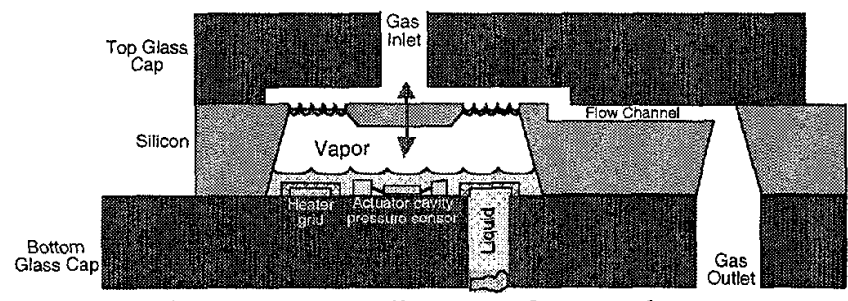

Figure 1. Thermopneumatically-actuated microvalve structure.

Deflection force is generated by the vapor pressure of the heated working fluid, which can be a volatile solvent such as pentane or methanol. Because a saturated liquid-vapor phase exists in the cavity, temperature determines the vapor pressure regardless of fill level. Earlier work by Bergstrom [2] demonstrated that the elimination of excess working fuid decreases system thermal mass and improves transient response.

The heater grids and integral capacitive pressure sensor (for real-time monitoring of actuator cavity pressure) are fabricated in a $2^{\text {nd }}$ Si layer bonded to the glass substrate. Elevating the heater grids above the glass substrate decreases steady-state power consumption by reducing direct heat loss to the substrate. Each of four heater grids consists of a lattice of $100 \mu \mathrm{m}$-long beam segments forming diamond-shaped pores, and each beam has a $4 \mu \mathrm{m}$-wide cross-section, shown in Figure 2. A $0.7 \mu \mathrm{m}$-thick polysilicon layer is used for the actual heating element (rather than the heavily boron-doped bulk Si grid frame) in order to allow independent control of the heater resistance by varying the polysilicon doping level. (The grid frame doping is fixcd at $>10^{20} \mathrm{~cm}^{-3}$ to ensure an adequate etch stop.) The dielectrics electrically isolate the poly-Si and protect it from attack during the final EDP wafer-dissolution step.

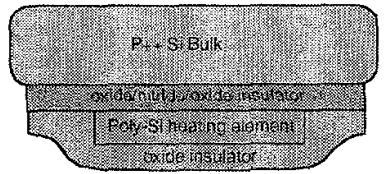

Figure 2. Cross-section of a beam in the heater grid.

A modified version of the dissolved-wafer process developed by Chavan for vacuum-sealed pressure sensors [3] is used to simultaneously fabricate the heater grids and pressure sensors on the glass substrate. Chavan's work gives an in-depth analysis of capacitive pressure sensor dynamics. Table 1 presents the design parameters used for the actuator pressure sensor in this work.

"Collin Rich is now with Integrated Sensing Systems (ISSYS), Inc., Ypsilanti, MI 48197. 
Table 1: Design parameters for actuator pressure sensor.

\begin{tabular}{lccccc} 
Webbing thickness & $2.4 \mu \mathrm{m}$ & \multicolumn{2}{c}{$\begin{array}{c}\text { Boss/electrode radius } \\
\text { Outer radius }\end{array}$} & $\begin{array}{c}124 \mu \mathrm{m} \\
250 \mu \mathrm{m}\end{array}$ \\
& $\begin{array}{ccccc}\text { Pressure } \\
\text { [torr] }\end{array}$ & $\begin{array}{c}\text { Deflection } \\
{[\mu \mathrm{m}]}\end{array}$ & $\begin{array}{c}\text { Gap } \\
{[\mu \mathrm{m}]}\end{array}$ & $\begin{array}{c}\text { Capacitance } \\
{[\mathrm{pF}]}\end{array}$ & $\begin{array}{c}\text { Sensitivity } \\
{[\mathrm{fF} / \mathrm{psi}]}\end{array}$ \\
\hline Min: & 760 & 2.2 & 6.8 & 0.0625 & 1.4 \\
Max: & 3000 & 8.7 & 0.3 & 1.4162 & 701.0 \\
\hline
\end{tabular}

A $\mathrm{KOH}$ recess step forms the heater grid posts and pressure sensor rim in the $2^{\text {nd }} \mathrm{Si}$, followed by boron etch stops to define the heater grid and pressure sensor bulk profiles. CVD polysilicon sandwiched between CVD dielectrics forms the active heating element in the grids, along with the sensor and grid lead transfers. The $2^{\text {nd }} \mathrm{Si}$ is then wafer-level anodically bonded to a metallized lower glass substrate. Finally, an EDP etch-back step dissolves the wafer bulk, leaving the pressure sensors and heater grids bonded to the glass substrate. The entire device is assembled with two additional anodic bonds, after which the cavities are insulated with $3 \mu \mathrm{m}$ of vapor-phase-deposited parylene, partially filled with pentane, and sealed. Parylene, which has very low thermal conductivity $(\mathrm{k}=0.0837 \mathrm{~W} / \mathrm{mK}$ ), reduces heat loss from the working fluid to the surrounding cavity.

\section{THERMAL MODELING}

A thermal model was developed for the valve structure. The fabricated device has been designed to facilitate an 8-bit microflow controller architecture, in conjunction with a separate microcontroller. It includes eight individually-valved, binarily weighted flow-metering channels in parallel, along with inlet and outlet pressure sensors. A steady-state model was derived from device geometry and material parameters to determine the thermal resistance between an energized actuator and the surrounding ambient. Conduction was assumed in the interior and through the lower substrate surface (i.e., mounting surface), whereas convection was assumed from the top and sides of the device (see Fig 3.)

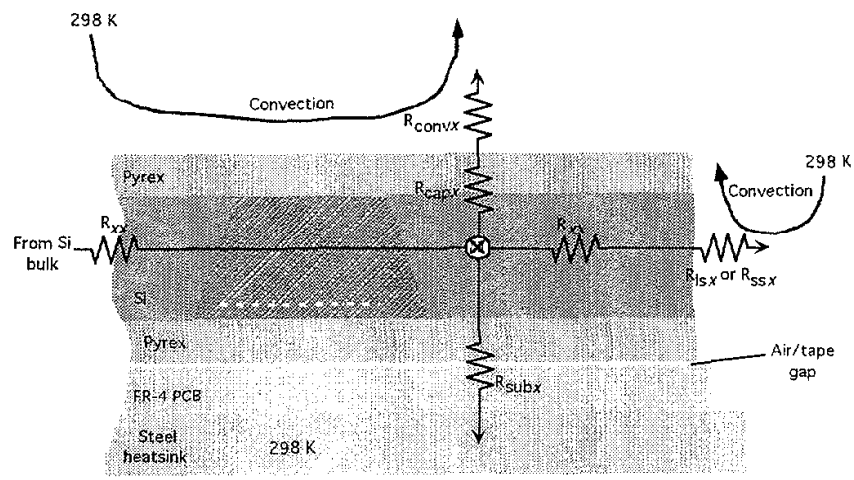

Figure 3. Simplified bulk thermal circuit for an analytical segment (including a non-energized actuator).

The steady-state thermal resistive network for the entire device is shown in Fig. 4. Simplifying the network and reducing the thermal mass of the system to a lumped-parameter model yields the final energized cavity model of Figure 5 . Similar analysis shows that heat loss through the grid posts is negligible compared to dissipation into the working fluid.

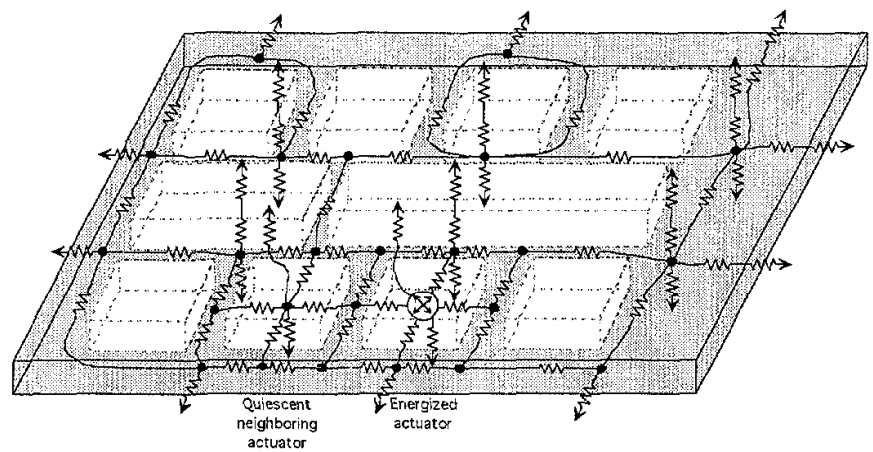

Figure 4. Thermal network for entire device. The eight small cavities are actuators. The two larger cavities contain the inlet and outlet pressure sensors.

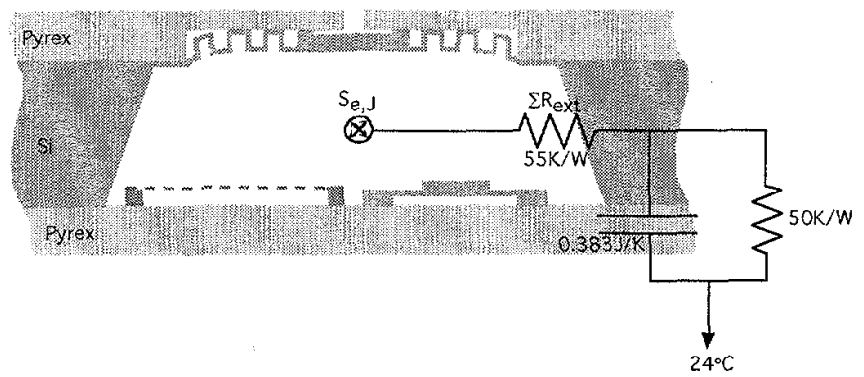

Figure 5. Lumped thermal model for energized actuator. Model relates input power to cavity temperature over time.

The Clausius-Clapeyron relation may be used to predict the pressure $p_{g}$ of an actuator cavity at temperature $T_{i g}$ (all temperatures in $\mathrm{K}$ ), assuming the cavity contains working fluid in both liquid and gaseous states (i.e. the fluid is saturated). This relation may be cast in integrated form [4] as:

$$
p_{g}=p_{g, 0} \exp ^{-\frac{M \Delta h_{r g}}{R_{g}}\left(\frac{1}{T_{l g}}-\frac{1}{T_{i g, a}}\right)} .
$$

An additional partial pressure term must also be added to account for residual air (an ideal gas) in the cavity. After substituting appropriate parameters for pentane, simulations yield an expected response as shown in Fig. 6.

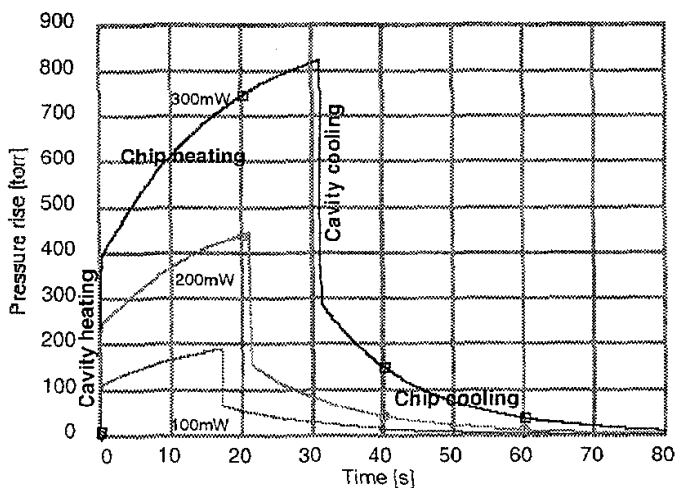

Figure 6 Simulated transient pressure vs. time for device mounted on semi-insulating substrate, at varied power levels. 


\section{EXPERIMENTAL RESULTS}

An assembled device along with an EDP-released lower substrate is shown in Figure 7. Figure 8 shows a SEM crosssection of a corrugated diaphragm, and Fig. 9 is a close-up of an actuator pressure sensor and heater grid cluster.

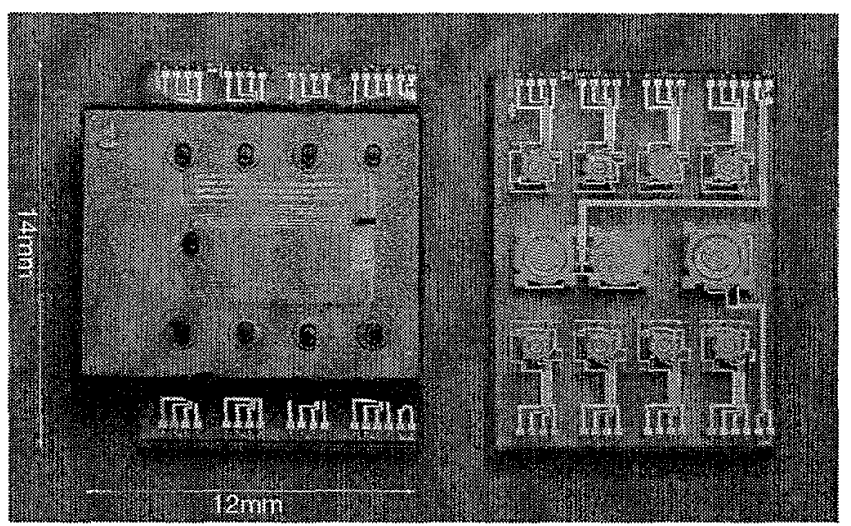

Figure 7. Left: assembled die with microvalves, flow channels, and pressure sensors for $\mu F C$ configuration; Right: lower substrate with heater grids and pressure sensors.

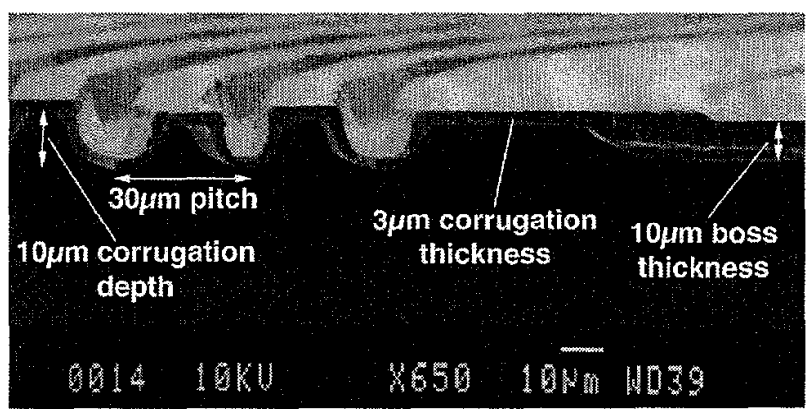

Figure 8. Cross-section of corrugations in valve diaphragm.

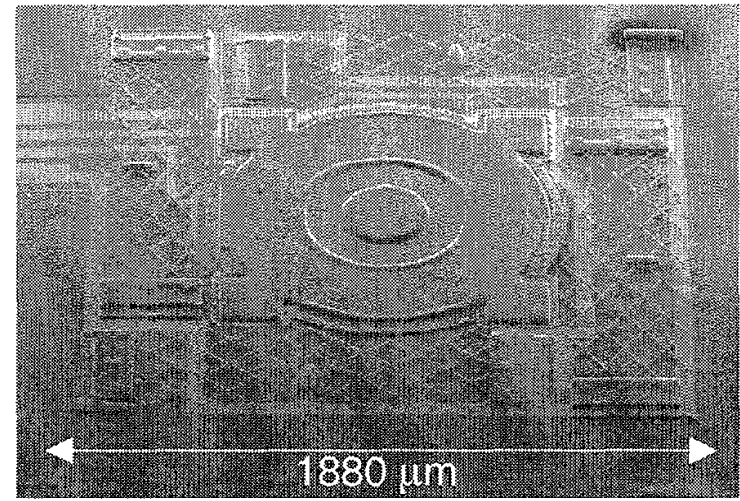

Figure 9. SEM of sensorfactuator cluster.

Flow rates for the structure varied linearly (flow was sonically limited) from 60-420sccm over a 700-1500torr range. Typical leak rates were $0.005-0.01 \mathrm{sccm}$, giving a dynamic range of $4 \times 10^{4}$ at 1500torr. A set of seven diaphragms was also pulsed with $15 \mathrm{psi}$ for one million cycles to test long-term reliability. No failures occurred.

Figures 10-13 plot actuator performance for several devices mounted on an insulating FR-4 (PCB) base, in room air ambient.

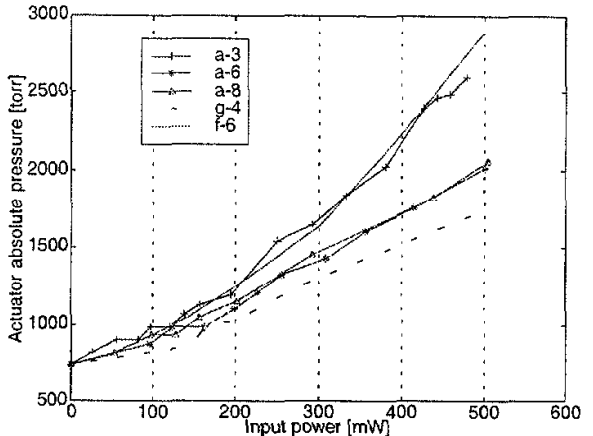

Figure 10. Steady-state pressure vs. power for pentane-filled actuators on insulating base.

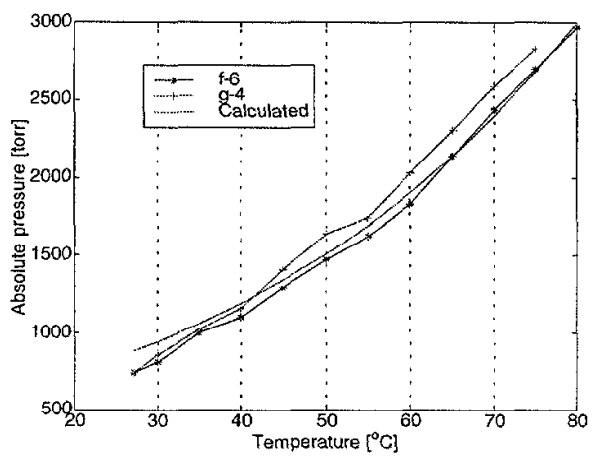

Figure 11. Steady-state pressure vs. temperature for pentanefilled actuators in temperature-controlled oven. Model prediction is also plotted.

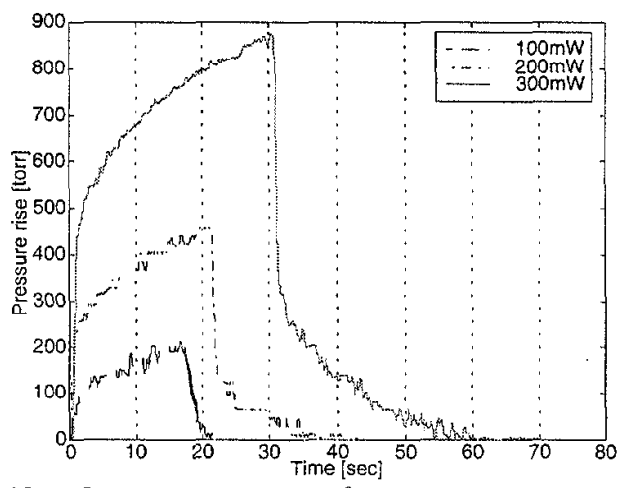

Figure 12. Transient response for one actuator on thermallyinsulating base.

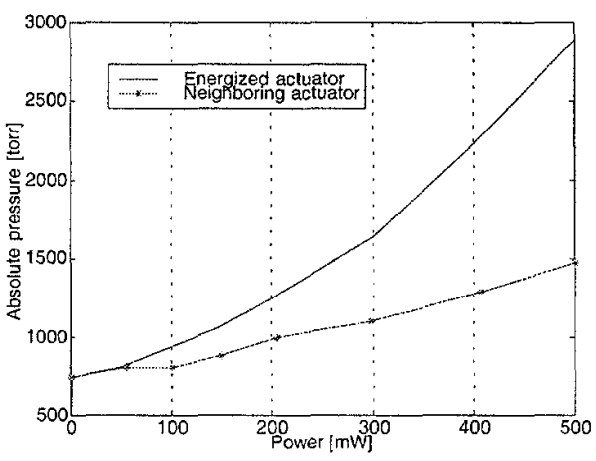

Figure 13. Cross-talk measurement: Steady-state pressure of "quiescent" actuator vs. power of neighboring, energized" actuator: Pressure of energized actuator is also shown. 
Table 2 summarizes the power performance of a valve in-situ at 1000 torr inlet, venting to vacuum. With the substrate exposed to vacuum, heat loss diminishes dramatically.

Table 2. Valve performance at 1000torr inlet to vacuum.

\begin{tabular}{cccc} 
Power $[\mathrm{mW}]$ & State & Flow $[\mathrm{sccm}]$ \\
\hline 0 & Open & 89 \\
350 & Closing & - \\
30 & Closed & .034 \\
50 & Closed & .005
\end{tabular}

\section{DESIGN OPTIMIZATION}

Analysis of modeling and results suggests the optimized structure of Fig. 14, which would require $5-10 \%$ as much power to achieve the same performance. Features of the redesign include: minimizing the glass and silicon thermal masses (decreases substrate heat storage); surrounding the actuator cavity with an airor vacuum-filled insulating buffer (decreases heat storage in rest of chip bulk and reduces crosstalk); and increasing the thickness of the insulating coating in the cavity (reduces bulk heat storage). Closure response time can also be improved with a closed-loop, spike-and-hold control scheme: quickly charge the thermal mass of the substrate with a high power spike, and then reduce power to maintain target pressure once the substrate equilibrates. An integrated pressure sensor is essential to such closed-loop control

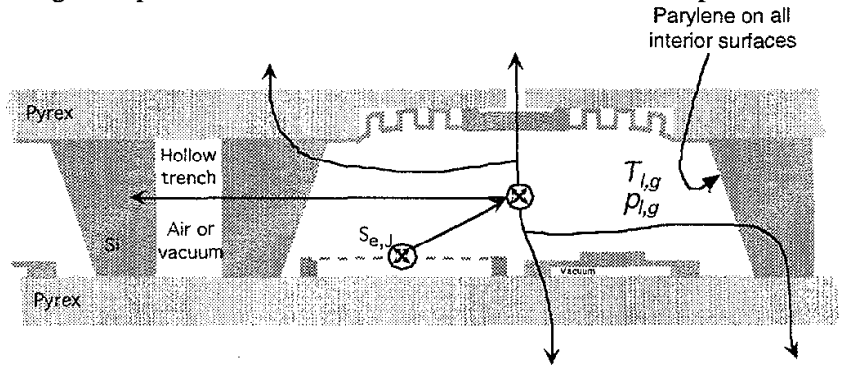

Figure 14. Revised valve structure for improved performance.

Simulation of the redesigned actuator predicts the response of Fig. 15. Table 3 compares the redsign to the experimental device. Furthermore, Fig. 16 shows the design space for the redesigned actuator. Device-to-ambient thermal resistance is the "tuning" factor: cooling time increases with resistance. From Fig. 16, a 2000 torr pressure rise with $50 \mathrm{~mW}$ input and a $1 \mathrm{sec}$ response time should be achievable.

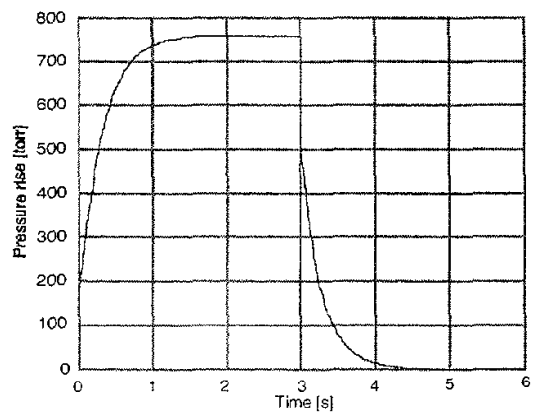

Figure 15. Simulated pressure response for the redesigned actuator with substrate mount similar to the fabricated device. input power is $155 \mathrm{~m} W$. Ambient temperature is $24^{\circ} \mathrm{C}$.
Table 3. Existing and redesigned actuator performance.

\begin{tabular}{lcc} 
Parameter & Existing & Redesign \\
\hline Example pressure rise & 750 torr & 750 torr \\
$90 \%$ rise/fall time & $15 \mathrm{sec}$ & $500 \mathrm{~ms}$ \\
Required power & $300 \mathrm{~mW}$ & $155 \mathrm{~mW}$
\end{tabular}

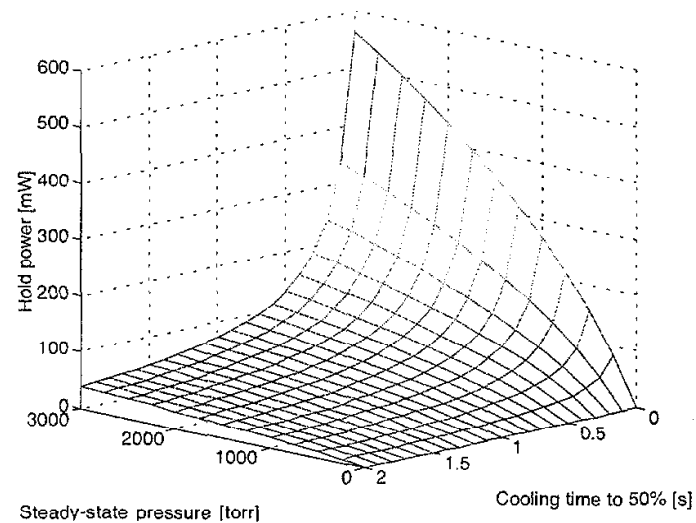

Figure 16. Required hold power vs. pressure rise and cooling (response) time for optimized actuator model. Cooling decay time is to $50 \%$ of steady-state pressure and is determined by thermat resistance of mount. $T_{f}^{*}=24^{\circ} \mathrm{C}$.

\section{CONCLUSIONS}

A thermopneumatic microvalve structure was designed, fabricated and tested. The structure demonstrated the feasibility of using raised heater grids and partially filled cavities to improve performance. The mechanical aspects of the valve (e.g. valve diaphragm) show good prospects for large dynamic range and long-term reliability. Real-time monitoring of actuator pressure was facilitated with integrated capacitive pressure sensors.

A model was also developed to predict pertormance for an optimized valve structure. The components of the model yield a good fit to experimental data. A redesigned actuator should achieve faster performance with an order of magnitude reduction in power consumption.

\section{ACKNOWLEDGEMENTS}

The authors would like to thank Prof. M. Kaviany for assistance with thermal modeling and Dr. A. Chavan for help with pressure sensor design.

\section{REFERENCES}

1. C. Rich and K. Wise, "An 8-bit Microflow Controller Using Pneumatically-Actuated Microvalves," Proc. 12 th Int'l. Conf. Microelectromechanical Systems, Orlando, FL, 1/99, IEEE (1999), pp. 130-134

2. P. Bergstrom, et al., "Thermally Driven Phase-Change Microactuation," J. Microelect. Systemts, 4, 10-17 (1995).

3. A. Chavan and K. Wise, "A Batch-Processed Vacuum-Sealed Capacitive Pressure Sensor," Proc. 1997 Int'l. Conf. on Solid-State Sensors and Actuators, 6/97, pp. 1449-1452.

4. M. Kaviany, Principles of Heat Transfer, Ann Arbor, MI, Fall 1999, pp. 717.718 